\title{
Independent electrons model for open quantum systems: Landauer-Büttiker formula and strict positivity of the entropy production
}

\author{
Gheorghe Nenciu \\ Dept. Theor. Phys., Univ. of Bucharest \\ P.O. Box MG 11, RO-077125, Bucharest, Romania \\ and \\ Institute of Mathematics of the Romanian Academy \\ PO Box 1-764, RO-014700 Bucharest, Romania \\ E-mail Gheorghe.Nenciu@imar.ro
}

\begin{abstract}
A general argument leading from the formula for currents through an open noninteracting mesoscopic system given by the theory of nonequilibrium steady states (NESS) to the Landauer-Büttiker formula is pointed out. Time reversal symmetry is not assumed. As a consequence it follows that, as far as the system has a nontrivial scattering theory and the reservoirs have different temperatures and/or chemical potentials, the entropy production is strictly positive.
\end{abstract}




\section{Introduction}

Landauer-Büttiker type formulas i.e. expressions relating the (charge, energy etc) currents through mesoscopic systems connected with electron reservoirs to the corresponding transmission coefficients have been proved to be a key tool for analyzing the quantum conductance in nanostructures. Obtained initially for the stationary case by phenomenological arguments [9], 8], 15] they have been widely extended and used. As for the derivation, one usually assumes that the reservoirs have a lead geometry and in order to make use of the asymptotic form of the scattered state the current is evaluated far away from the scatterer [7], 11], 18, a procedure justified (at least in the stationary regime) by charge conservation. However this approach may become problematic for other reservoir geometries when the leads are short or even inexistent (see e.g. [14, 6]) or in non-stationary regime.

At a more basic level one starts from a non-equilibrium statistical mechanics formulation (e.g. linear response theory, NESS theory etc) and the problem of proving the Landauer-Büttiker formula is to show that the obtained formula for the current can be cast in a form in which the structure of the mesoscopic system enters only via its transition matrix of the associated scattering problem as suggested by the phenomenological derivation. In this note we shall consider the formula for the current as given by NESS theory (see e.g [22, [12, 2], 16] and references therein). One starts at $t=0$ from an equilibrium state of the decoupled system (i.e. no coupling between the mesoscopic system and the reservoirs) with reservoirs having different temperatures and/or chemical potentials. At the one particle level the system is described by

$$
H_{0}=H_{\mathcal{S}}+\sum_{j=1}^{N} H_{j} ; \mathcal{H}=\mathcal{H}_{\mathcal{S}} \oplus_{j=1}^{N} \mathcal{H}_{j}
$$

where $\mathcal{H}_{\mathcal{S}}, H_{\mathcal{S}}$ are the Hilbert space and hamiltonian of the mesoscopic system and $\mathcal{H}_{j}, H_{j}$ are the Hilbert space and the hamiltonian of the jth reservoir. The coupling, described at the one particle level by $V$, is switched on suddenly at $t=0$. In the limit $t \rightarrow \infty$ the system settles down to a nonequilibrium stationary state. The currents out from the reservoirs are defined as minus the time variation of their charge. Since the electrons are considered independent the second quantization machinery allows to write the currents as given by the general NESS theory in terms of one-particle objects. More precisely if $\beta_{j}, \mu_{j}$ are the temperature and the chemical potential respectively of the jth reservoir in the initial state then the current out from the kth reservoir in the "final" steady state is [2] (the charge of the electron is $-e$ ):

$$
j_{k}=i e \operatorname{Tr}_{\mathcal{H}}\left(\Omega_{+} \Pi_{0} F_{0} \Pi_{0} \Omega_{+}^{*}\left[V, \Pi_{k}\right]\right)=i e \operatorname{Tr}_{\mathcal{H}}\left(\Pi_{0} F_{0} \Pi_{0} \Omega_{+}^{*}\left[V, \Pi_{k}\right] \Omega_{+}\right)
$$


where $\Pi_{j}$ are the orthogonal projections onto $\mathcal{H}_{j}$ in $\mathcal{H}, \Pi_{0}=\sum_{j=1}^{N} \Pi_{j}$,

$$
\Omega_{+}=s-\lim _{t \rightarrow-\infty} e^{i t\left(H_{0}+V\right)} e^{-i t H_{0}} \Pi_{0}
$$

(we follow the notation in the physical literature and 21] ) and

$$
\Pi_{0} F_{0} \Pi_{0}=\sum_{j=1}^{N} f_{\beta_{j}, \mu_{j}}^{F D}\left(H_{j}\right) .
$$

Here $f_{\beta_{j}, \mu_{j}}^{F D}(x)$ are the usual Fermi-Dirac functions:

$$
f_{\beta_{j}, \mu_{j}}^{F D}(x)=\left(1+e^{\beta_{j}\left(x-\mu_{j}\right)}\right)^{-1} .
$$

Two remarks are in order here. Firstly, we would like to stress the fact that (1.2) gives only the steady part of the current; a (quasi) periodic component might also exists if $H=H_{0}+V$ has bound states (see e.g. [16] Section 5.5 for a discussion about this point). Secondly, due to the fact that there is no interaction between electrons, (1.2) can be obtained by means of elementary second quantization theory without the use of the heavy artilery of NESS theory.

In the framework of NESS theory and under appropriate technical conditions the case $\operatorname{dim} \mathcal{H}_{\mathcal{S}}=1$ (single state quantum dot) has been thoroughly studied in [2, [16]; in particular the Landauer-Büttiker formula has been proved and strict positivity of the entropy production established. Actually in this case the model is nothing but the well known exactly solvable Wigner-Weisskopf Atom model (known also under the name of Friedrichs model) for which the Møller operator as well as the scattering matrix can be explicitly written down so that the Landauer-Büttiker formula can be directly verified. Moreover an extension of the analysis in [2], [16] to more general models similar to the one considered in the present note will be given (C.-A. Pillet: private communication) in [3] announced in [2].

An alternative way (and sometimes more satisfactory from the physical point of view) of computing currents in non-equilibrium statistical mechanics is to start at $t=-\infty$ with reservoirs at the same temperature and chemical potential and with an equilibrium state of the coupled system and then switch on adiabatically the bias in chemical potential and/or temperature. Unfortunately due to the fact that in this case the "perturbation" is not localized the problem is much more difficult and it has been worked out only at the linear response theory level. In this context the Landauer-Büttiker formula has been shown to hold true at the heuristic level by Baranger and Stone [7] and rigorously proved for a tight-binding model for reservoirs by Cornean, Jensen and Moldoveanu [10]. 
Coming back to the formula (1.2) the problem is that the Møller operator, $\Omega_{+}$, involves only "half" of the evolution from $-\infty$ to $\infty$ encoded in the scattering matrix so one has to show that one can rewrite the current only in terms of scattering matrix and the initial equilibrium state. In the related context of adiabatic quantum pumps theory it has been proved in [5] that this is indeed the case for the lead geometry of the reservoirs.

The aim of this note (which is a revision and extension of [20]) is a narrow one: to outline a general argument leading from (1.2) to the LandauerBüttiker formula. The argument is entirely elementary and very general: it works for an arbitrary geometry of the reservoirs (e.g. half spaces, semi infinite leads with arbitrary section etc) and arbitrary mesoscopic systems of finite size. Also we allow the reservoirs to be coupled both via the mesoscopic system and by direct contacts [12]. Actually, the only thing which is needed is a good stationary scattering theory for the pair $\left(H_{0}, H_{0}+V\right)$. This is consistent with the generality of the phenomenological arguments leading to Landauer-Büttiker formula. For the model at hand the strict positivity of the entropy (which is a central issue in NESS theory and has been established under various conditions for by far more general models see e.g. 2], [4], [19] and references therein) follows from the Landauer-Búttiker formula; while for systems with time reversal symmetry this is straightforward by the argument in [2] in the general case the argument is a bit more involved.

The content of the note is as follows. In Section 2 we specify the model and give the needed formulas from stationary scattering theory. Section 3 contains the argument leading from the formula (1.2) to the LandauerBüttiker formula. In order not to burden the simplicity of the argument in technical and notational details we shall give it at the formal level and in a simple context: two reservoirs with the same simple (single channel) absolutely continuous spectrum, $\sigma_{0} \subset[0, \infty)$, and mesoscopic systems with a finite number of states (i.e. $\operatorname{dim} \mathcal{H}_{\mathcal{S}}=M<\infty$ ). For a mathematical substantiation for various concrete models one has to make precise the technical conditions on $H_{0}$ and $V$ and then check that one can apply the results of the rigorous stationary scattering theory as developed e.g. in [1], 24], 25] (for stationary scattering theory at the formal level we send the reader to [13]). In Section 4 we give some straightforward extensions as well as the argument for strict positivity of the entropy production. 


\section{The model and its scattering theory}

As already said in the introduction, at the one particle level the uncoupled system is described by

$$
H_{0}=H_{\mathcal{S}}+\sum_{j=1}^{N} H_{j} ; \mathcal{H}=\mathcal{H}_{\mathcal{S}} \oplus_{j=1}^{N} \mathcal{H}_{j}
$$

where $\mathcal{H}_{\mathcal{S}}, H_{\mathcal{S}}$ are the Hilbert space and hamiltonian of the mesoscopic system and $\mathcal{H}_{j}, H_{j}$ are the Hilbert space and the hamiltonian of the jth reservoir. We suppose that the spectral representation of $H_{j} j=1,2$ is given in terms of generalized eigenfunctions, $\left|\psi_{j, E}^{0}\right\rangle$, living in an appropriate "weighted" Hilbert space, $\mathcal{K}_{j}^{*}$ (Gelfand triplets structure: $\mathcal{K}_{j} \subset \mathcal{H}_{j} \subset \mathcal{K}_{j}^{*}$ ) :

$$
H_{j}\left|\psi_{j, E}^{0}>=E\right| \psi_{j, E}^{0}>, E \in \sigma\left(H_{j}\right)=\sigma_{0} \subset[0, \infty)
$$

For $f \in \mathcal{H}_{j}$ we denote by $f(E)$ its generalized Fourier transform:

$$
f(E)=<\psi_{j, E}^{0}, f>.
$$

As concerning $V$, we suppose to have the following structure (in the decomposition given by (1.1)):

$$
V=\left(\begin{array}{ccc}
0 & V_{\mathcal{S} 1} & V_{\mathcal{S} 2} \\
V_{\mathcal{S} 1}^{*} & 0 & V_{12} \\
V_{\mathcal{S} 2}^{*} & V_{12}^{*} & 0
\end{array}\right)
$$

Since $\mathcal{H}_{\mathcal{S}}$ has finite dimension, $V_{\mathcal{S} 1}$ and $V_{\mathcal{S} 2}$ are finite rank operators. We suppose the "direct contact", $V_{12}$, to be also of finite rank. Accordingly:

$$
\begin{aligned}
V_{\mathcal{S} j} & =\sum_{l=1}^{m_{j} \leq M} v_{j l}\left|s_{j l}><f_{j l}\right|, \\
V_{12} & =\sum_{l=1}^{m<\infty} v_{l}\left|g_{1 l}><g_{2 l}\right|
\end{aligned}
$$

where $j=1,2,\left\{f_{j l}\right\}_{l=1}^{m_{j}},\left\{g_{j l}\right\}_{l=1}^{m},\left\{s_{j l}\right\}_{l=1}^{m_{j}}$ are orthonormal systems in $\mathcal{H}_{j}$ and $\mathcal{H}_{\mathcal{S}}$ respectively and $v_{j l}, v_{l}>0$.

Since $V$ is of finite rank, by Kato-Kuroda-Birman theory [21], 24], 25] the Møller operators

$$
\Omega_{ \pm}=s-\lim _{t \rightarrow \mp \infty} e^{i t\left(H_{0}+V\right)} e^{-i t H_{0}} \Pi_{0}
$$


exist and are unitary from $\Pi_{0} \mathcal{H}$ onto the absolutely continuous subspace, $\Pi_{a c} \mathcal{H}$, of $H$.

We impose further conditions on $f_{j l}, g_{j l}$ in order to assure that $\Omega_{ \pm}$provide spectral representations for $H$ restricted to $\Pi_{a c} \mathcal{H}$ ie for all $E \in \sigma_{a c}(H)=$ $\cup_{j=1}^{n} \sigma\left(H_{j}\right)$, with a possible exception of a discrete set, $\mathcal{E}, \Omega_{ \pm}$have bounded extensions in the orthogonal sum of $\mathcal{K}_{j}^{*}$ and

$$
\left|\psi_{j, E}^{ \pm}>=\Omega_{ \pm}\right| \psi_{j, E}^{0}>
$$

are generalized eigenfunctions for $H$ :

$$
H\left|\psi_{j, E}^{ \pm}>=E\right| \psi_{j, E}^{ \pm}>
$$

A sufficient condition (which at the price of more technicalities can be weakened) in the case when $H_{j}$ are discrete or continuous Laplaceans supplemented with boundary conditions is that $f_{j l}, g_{j l}$ are exponentially localized in space. This condition also implies that the generalized Fourier coefficients, $f_{j l}(E), g_{j l}(E)$ (see (2.3) ) of $f_{j l}, g_{j l}$ are smooth functions of $E$, a fact which is needed in order to apply the principal value formula during the proof below. The generalized eigenfunctions satisfy the Lippmann-Schwinger [13, [1], 24], 25] equation:

$$
\left|\psi_{j, E}^{ \pm}>=\right| \psi_{j, E}^{0}>-\left(H_{0}-E \mp i 0\right)^{-1} V \mid \psi_{j, E}^{ \pm}>\text {. }
$$

Consider now the scattering operator

$$
S=\Omega_{-}^{*} \Omega_{+}
$$

and the corresponding transition operator, $T$, defined by

$$
S=1-2 i \pi T \text {. }
$$

Since $S$ (and then $T$ ) commutes with $H_{0}$ it has a spectral representation:

$$
S=\int_{\sigma_{0}} S(E) d E, \quad T=\int_{\sigma_{0}} T(E) d E
$$

where $S(E)$ is a unitary two by two matrix (we are considering the case of two reservoirs with simple spectrum). From the unitarity of $S(E)$ it follows that $T(E)$ satisfies the so called optical theorem:

$$
T(E)-T^{*}(E)=-2 \pi i T(E) T^{*}(E) .
$$

The basic result of the stationary scattering theory is the formula for $T(E)$ in terms of the generalized eigenfunctions of $H_{0}$ [13], [1], 24], 25]:

$$
T_{j k}(E)=<\psi_{j, E}^{0}, V \psi_{k, E}^{+}>=<\psi_{j, E}^{0}, V \Omega_{+} \psi_{k, E}^{0}>.
$$




\section{Landauer-Büttiker formula}

To prove the Landauer-Büttiker formula in the context described above amounts to prove :

\section{Proposition 1.}

$$
\begin{gathered}
j_{1}=i e \operatorname{Tr}_{\mathcal{H}}\left(\Omega_{+} \Pi_{0} F_{0} \Pi_{0} \Omega_{+}^{*}\left[V, \Pi_{1}\right]\right)= \\
-2 e \pi \int_{\sigma_{0}} d E\left(f_{\beta_{1}, \mu_{1}}^{F D}(E)-f_{\beta_{2}, \mu_{2}}^{F D}(E)\right)\left|T_{12}(E)\right|^{2} .
\end{gathered}
$$

The second equality in (3.1) is the main result of this note.

We compute $j_{1}$ from (1.2). Inserting the formula for $V$ (see (2.4), (2.5), (2.6) ) and computing the trace in appropriate bases one gets:

$$
\begin{gathered}
j_{1}=-2 e \Im\left(\sum_{l=1}^{l=m_{1}} v_{1 l}<f_{1 l}, \Omega_{+} \Pi_{0} F_{0} \Pi_{0} \Omega_{+}^{*} s_{1 l}>+\right. \\
\left.\sum_{l=1}^{l=m} v_{l}<g_{1 l}, \Omega_{+} \Pi_{0} F_{0} \Pi_{0} \Omega_{+}^{*} g_{2 l}>\right) .
\end{gathered}
$$

Using the spectral representation of $\Pi_{0} F_{0} \Pi_{0}$ (see (1.4) ) in (3.2) or, alternatively, evaluating directly the trace in the r.h.s. of (1.2) in the generalized basis of $H_{0}$ one gets:

$$
\begin{gathered}
j_{1}=-2 e \int_{\sigma_{0}} d E\left\{f_{\beta_{1}, \mu_{1}}^{F D}(E) \Im<V \Omega_{+} \psi_{1, E}^{0}, \Pi_{1} \Omega_{+} \psi_{1, E}^{0}>+\right. \\
\left.f_{\beta_{2}, \mu_{2}}^{F D}(E) \Im<V \Omega_{+} \psi_{2, E}^{0}, \Pi_{1} \Omega_{+} \psi_{2, E}^{0}>\right\} .
\end{gathered}
$$

Let us compute first the coefficient of $f_{\beta_{1}, \mu_{1}}^{F D}(E)$ in (3.3). Using the LippmannSchwinger equation (see (2.10) ) for $\Omega_{+} \psi_{1, E}^{0}$ and the spectral representation of $H_{0}$ one has:

$$
\begin{gathered}
<V \Omega_{+} \psi_{1, E}^{0}, \Pi_{1} \Omega_{+} \psi_{1, E}^{0}>= \\
<V \Omega_{+} \psi_{1, E}^{0}, \psi_{1, E}^{0}>-<V \Omega_{+} \psi_{1, E}^{0}, \Pi_{1} \frac{1}{H_{0}-E-i 0} V \Omega_{+} \psi_{1, E}^{0}>= \\
\frac{T_{11}(E)}{-\int d E^{\prime} \frac{\left|<\psi_{1, E^{\prime}}^{0}, V \Omega_{+} \psi_{1, E}^{0}>\right|^{2}}{E^{\prime}-E-i 0}} .
\end{gathered}
$$

Now the important fact is that we need only the imaginary part of (3.4). Then using the principal value formula

$$
\frac{1}{x-i 0}=i \pi \delta(0)+P V \frac{1}{x}
$$

to evaluate the integral in (3.4) ( since $\left|<\psi_{1, E^{\prime}}^{0}, V \Omega_{+} \psi_{1, E}^{0}>\right|^{2}$ depends upon $E^{\prime}$ only via $f_{1 l}\left(E^{\prime}\right)$ and $g_{1 l}\left(E^{\prime}\right)$ which are smooth by assumption, this is legitimate) one obtains that the coefficient of $f_{\beta_{1}, \mu_{1}}^{F D}(E)$ in (3.3) is

$$
\Im\left(\overline{T_{11}(E)}-i \pi\left|T_{11}(E)\right|^{2}\right) .
$$

Now the use of the optical theorem (2.14) leads to the conclusion that the coefficient of $f_{\beta_{1}, \mu_{1}}^{F D}(E)$ in $(3.3)$ is $\pi\left|T_{12}(E)\right|^{2}$. A similar computation for the coefficient of $f_{\beta_{2}, \mu_{2}}^{F D}(E)$ in (3.3) (in this case the term linear in $T(E)$ vanishes) leads to $-\pi\left|T_{12}(E)\right|^{2}$ and the proof of (3.1) is finished. 


\section{Generalizations and strict positivity of the entropy production}

We give first some straightforward extensions of the result in previous section.

i. A similar proof applied to the energy current (see e.g. 2]) gives:

$$
\begin{aligned}
& \Phi_{1}=-i \operatorname{Tr}_{\mathcal{H}}\left(\Omega_{+} \Pi_{0} F_{0} \Pi_{0} \Omega_{+}^{*}\left[V, \Pi_{1} H_{0} \Pi_{1}\right]\right)= \\
& 2 \pi \int_{\sigma_{0}} d E\left(f_{\beta_{1}, \mu_{1}}^{F D}(E)-f_{\beta_{2}, \mu_{2}}^{F D}(E)\right) E\left|T_{12}(E)\right|^{2} .
\end{aligned}
$$

ii. The condition that $\sigma\left(H_{1}\right)=\sigma\left(H_{2}\right)$ (as sets) is not necessary ; in the general case only the energies in the intersection of $\sigma\left(H_{1}\right)$ with $\sigma\left(H_{2}\right)$ can have nontrivial scattering and then contribute to the current.

iii. The straightforward generalization of (3.1), (4.11) to the case of $N$ reservoirs is given by:

$$
\begin{gathered}
j_{k}=i e \operatorname{Tr}_{\mathcal{H}}\left(\Omega_{+} \Pi_{0} F_{0} \Pi_{0} \Omega_{+}^{*}\left[V, \Pi_{k}\right]\right)= \\
-2 e \pi \int_{\sigma_{0}} d E \sum_{j=1}^{N}\left(f_{\beta_{k}, \mu_{k}}^{F D}(E)-f_{\beta_{j}, \mu_{j}}^{F D}(E)\right)\left|T_{k j}(E)\right|^{2}, \\
\Phi_{k}=-i \operatorname{Tr}_{\mathcal{H}}\left(\Omega_{+} \Pi_{0} F_{0} \Pi_{0} \Omega_{+}^{*}\left[V, \Pi_{k} H_{0} \Pi_{k}\right]\right)= \\
2 \pi \int_{\sigma_{0}} d E \sum_{j=1}^{N}\left(f_{\beta_{k}, \mu_{k}}^{F D}(E)-f_{\beta_{j}, \mu_{j}}^{F D}(E)\right) E\left|T_{k j}(E)\right|^{2} .
\end{gathered}
$$

Notice that for $N>2$ and systems without time reversal symmetry one can have $\left|T_{k j}(E)\right|^{2} \neq\left|T_{j k}(E)\right|^{2}$. Still $\sum_{k=1}^{N} j_{k}=0, \sum_{k=1}^{N} \Phi_{k}=0$ as required by charge and energy conservation, due to the fact that the unitarity of $S$ implies that $T(E)$ is a normal matrix:

$$
T(E) T^{*}(E)=T^{*}(E) T(E)
$$

iv. If at some energy $E$ the spectra of $H_{j}$ do not have multiplicity one then $T_{j k}(E)$ become operators $T_{j k}(E): \mathcal{H}_{j}(E) \rightarrow \mathcal{H}_{k}(E)$ and $\left|T_{j k}(E)\right|^{2}$ in (4.2) are to be replaced by $\operatorname{Tr}_{\mathcal{H}_{j}(E)} T_{j k}(E) T_{j k}^{*}(E)$.

Consider now the entropy production. The entropy production rate (as given by heuristic thermodynamic arguments) has been identified as the (thermodynamic and $t \rightarrow \infty$ ) limit of the relative entropy of the evolved state with respect to the initial state, [23, [17, [12, 22], and then its positivity follows from Klein's inequality. However, although for finite reservoirs and times its strict positivity can be also easily obtained from Klein's inequality it is not clear whether it survives when taking thermodynamic and infinite time limits. Accordingly the strict positivity of the entropy production has to be established for each concrete realization of NESS theory and 
many results are known (see e.g. [4, [2, [19] and references therein). For the model considered in this note the entropy production, $\sigma$, writes as (see e.g. formula (2.61) in [12] or formula (6.46) in [2]):

$$
\sigma=-\sum_{k=1}^{N} \beta_{k}\left(\Phi_{k}-\mu_{k} \mathcal{I}_{k}\right)
$$

where

$$
\mathcal{I}_{k}=-\frac{1}{e} j_{k}=2 \pi \int_{\sigma_{0}} d E \sum_{j=1}^{N}\left(f_{\beta_{k}, \mu_{k}}^{F D}(E)-f_{\beta_{j}, \mu_{j}}^{F D}(E)\right)\left|T_{k j}(E)\right|^{2}
$$

is the particle current. From (4.8), (4.2) and (4.6) and using the notations:

$$
f(x)=(1+x)^{-1} ; \quad x_{E, j}=\beta_{j}\left(E-\mu_{j}\right)
$$

one obtains:

$$
\sigma=-2 \pi \int_{\sigma_{0}} d E \sum_{j, k=1}^{N}\left(f\left(x_{E, k}\right)-f\left(x_{E, j}\right)\right) x_{E, k}\left|T_{k, j}(E)\right|^{2} .
$$

Using

$$
\sum_{m=1}^{N}\left|T_{l, m}(E)\right|^{2}=\sum_{m=1}^{N}\left|T_{m, l}(E)\right|^{2}, \quad l=1,2, \ldots, N
$$

which follows from (4.4), one can rewrite (4.8) as

$$
\sigma=2 \pi \int_{\sigma_{0}} d E \sum_{j, k=1}^{N} f\left(x_{E, j}\right)\left(x_{E, k}-x_{E, j}\right)\left|T_{k, j}(E)\right|^{2}
$$

We shall say that the model has a nontrivial scattering in the channel $\left(j_{0}, k_{0}\right)$ if $T_{j_{0}, k_{0}}(E) \neq 0$ (on a subset of $\sigma_{0}$ of positive Lebesgue measure). As expected, the non-triviality of the scattering implies strict positivity of the entropy production:

Corollary 2. Suppose that the scattering is nontrivial in the channel $\left(j_{0}, k_{0}\right)$ and in addition $\left(\beta_{j_{0}}, \mu_{j_{0}}\right) \neq\left(\beta_{k_{0}}, \mu_{k_{0}}\right)$ (i.e. at least $\beta_{j_{0}} \neq \beta_{k_{0}}$ or $\left.\mu_{j_{0}} \neq \mu_{k_{0}}\right)$. Then

$$
\sigma>0
$$


Proof. For the time reversal symmetric case i.e. $\left|T_{l, m}(E)\right|=\left|T_{m, l}(E)\right|$ (4.11) follows from a simple argument in [2]: by symmetrizing the sum in either (4.8) or (4.10) one obtains:

$$
\sigma=\pi \int_{\sigma_{0}} d E \sum_{j, k=1}^{N}\left(f\left(x_{E, j}\right)-f\left(x_{E, k}\right)\right)\left(x_{E, k}-x_{E, j}\right)\left|T_{k, j}(E)\right|^{2}
$$

and then (4.11) follows from the fact that $f(x)$ is strictly decreasing. In the general case the argument is a bit more involved and, as expected, mimic the proof of Klein's inequality. Let $F(x)$ be a primitive of $f(x)$. Since $f(x)$ is strictly decreasing, $F(x)$ is strictly concave i.e

$$
F(x) \leq F(y)+f(y)(x-y)
$$

and the equality is reached only for $x=y$. From (4.13) and (4.9) it follows that

$$
\begin{gathered}
\sum_{j=1, k}^{N} f\left(x_{E, j}\right)\left(x_{E, k}-x_{E, j}\right)\left|T_{k, j}(E)\right|^{2} \geq \sum_{j, k=1}^{N}\left(F\left(x_{E, k}\right)-F\left(x_{E, j}\right)\left|T_{k, j}(E)\right|^{2}\right. \\
=\sum_{j, k=1}^{N} F\left(x_{E, k}\right)\left(\left|T_{k, j}(E)\right|^{2}-\left|T_{j, k}(E)\right|^{2}\right)=0
\end{gathered}
$$

and the equality is attained only if for all pairs $(j, k)$ and all (a.e) $E,\left(x_{E, k}-\right.$ $\left.x_{E, j}\right)\left|T_{k, j}(E)\right|=0$. Then the observation that $x_{E, k_{0}}-x_{E, j_{0}}=0$ holds true for at most one energy finishes the proof .

\section{Acknowledgments}

I would like to thank Claude-Alain Pillet for stimulating discussions about NESS theory in general and about the derivation of the Landauer-Büttiker formula in particular. This research has been supported by the CEEX Grant 05-D11-45/2005. This note has been partly written during a visit at Aalborg University. Both the financial support and the hospitality of the Department of Mathematical Sciences, Aalborg University, are gratefully acknowledged.

\section{References}

[1] W. O. Amrein, J. M. Jauch, K. B. Sinha: Scattering Theory in Quantum Mechanics, Benjamin, 1977.

[2] W. Aschbacher, V. Jaksic, Y. Pautrat, C.-A. Pillet: Topics in nonequilibrium statistical mechanics. mp_arc 05-207.

[3] W. Aschbacher, V. Jaksic, Y. Pautrat, C.-A. Pillet:Transport properties of ideal Fermi gases (in preparation). 
[4] W.Aschbacher, H. Spohn: A remark on the strict positivity of the entropy production. Lett. Math. Phys. bf 75, 17-23 (2006).

[5] J. E. Avron, A. Elgart, G-M. Graf, L. Sadun, K. Schnee: Adiabatic charge pumping in open quantum systems. Commun. Pure. Appl. Math. 57, 528-561 (2004).

[6] A. H. Barnett, M. Blaauboer, A. Mody, E. J. Heller: Mesoscopic scattering in the half plane: Squeezing conductance through a small hole. Phys. Rev. B 63, 245312 (2001).

[7] H.U. Baranger, A.D. Stone: Electrical linear response theory in an arbitrary magnetic field: A new Fermi-surface formation. Phys. Rev. B 40, 8169-8193 (1989).

[8] M. Büttiker: Four terminal phase-coherent conductance. Phys.Rev. Lett. 57, 1761-1764 (1986).

[9] M. Büttiker, Y. Imry, R. Landauer, S. Pinhas: Generalized manychannel conductance with application to small rings. Phys. Rev. B 31 6207-6215 (1985).

[10] H. D. Cornean, A. Jensen, V. Moldoveanu: A rigorous proof of the Landauer-Büttiker formula. J. Math. Phys. 46, 042106 (2005).

[11] D. S. Fisher, P.A. Lee: Relation between conductivity and transmission matrix. Phys. Rev. B 23, 6851-6854 (1981).

[12] J. Frölich, M. Merkli, D. Ueltschi: Dissipative transport: Thermal contacts and tunnelling junctions. Ann. Henri Poincaré 4, 897-945 (2003).

[13] M. L. Goldberger, K. M. Watson: Collision Theory, Snd Edition, Dover (2004).

[14] A. W. Holleitner, C. R. Decker, H. Qin, K. Eberl, R. H. Blick: Coherent coupling of two quantum dots embedded in an Aharonov-Bohm interferometer. Phys. Rev. Lett. 87 , 256802 (2001).

[15] Y. Imry, R. Landauer: Conductance viewed as transmission. Rev. Mod. Phys. 71, S306-S312 (1999).

[16] V. Jaksic, E. Kritchevski, C.-A. Pillet: Mathematical theory of the Wigner-Weisskopf Atom. mp_arc 05-333 (To appear in Lecture Notes in Mathematics, 2006). 
[17] V. Jaksic, C.-A. Pillet: On entropy production in quantum statistical mechanics. Comm. Math. Phys. 217, 285-293 (2001).

[18] P. Mavropoulos, N. Papanikolaou, P. H. Dederichs: Korringa-KohnRostoker formalism for ballistic transport. Phys. Rev. B 69, 125104 (2004).

[19] M. Merkli, M. Mück, I. M. Sigal Theory of non-equilibrium stationary states as a theory of resonances. Existence and properties of NESS. arXiv: math-ph 0603006 .

[20] G. Nenciu: A general proof of Landauer-Büttiker formula. arXiv: math-ph 0603030

[21] M. Reed and B. Simon: Methods of Modern Mathematical Physics. Vol. III. Scattering Theory, Academic Press, 1979.

[22] D. Ruelle: Natural non-equilibrium states in quantum statistical mechanics. J. Stat. Phys. 98, 57-75 (2000).

[23] D. Ruelle: Entropy production in quantum spin systems. Comm. Math.Phys. 224, 3-16 (2001).

[24] D. Yafaev: Scattering Theory: Some Old and New Problems, LNM 1735, Springer, 2000.

[25] D. Yafaev: Mathematical Scattering Theory, AMS, 1992. 